\title{
Histopathological and immunohistochemical findings of 20 autopsy cases with 2009 H1N1 virus infection
}

Noriko Nakajima ${ }^{1}$, Yuko Sato ${ }^{1}$, Harutaka Katano ${ }^{1}$, Hideki Hasegawa ${ }^{2}$, Toshio Kumasaka ${ }^{3}$, Satoru Hata ${ }^{4}$, Shinya Tanaka ${ }^{5}$, Tomonori Amano ${ }^{6}$, Takahiko Kasai ${ }^{7}$, Ja-Mun Chong ${ }^{8}$, Toshihiko Iiduka ${ }^{9}$, Iwao Nakazato ${ }^{10}$, Yohko Hino ${ }^{11}$, Akihiko Hamamatsu ${ }^{12}$, Hisashi Horiguchi ${ }^{13}$, Tomoyuki Tanaka ${ }^{14}$, Akio Hasagawa ${ }^{15}$, Yoshiaki Kanaya ${ }^{16}$, Reiko Oku ${ }^{17}$, Takeshi Oya ${ }^{18}$ and Tetsutaro Sata ${ }^{1}$

${ }^{1}$ Department of Pathology, National Institute of Infectious Diseases, Tokyo, Japan; ${ }^{2}$ Influenza Virus Research Center, National Institute of Infectious Diseases, Tokyo, Japan; ${ }^{3}$ Department of Pathology, Japanese Red Cross Medical Center, Tokyo, Japan; ${ }^{4}$ Department of Clinical Laboratory Sciences, Nagano Red Cross Hospital, Nagano, Japan; ${ }^{5}$ Laboratory of Cancer Research, Department of Pathology, Hokkaido University Graduate School of Medicine, Sapporo, Japan; ${ }^{6}$ Department of Cardiovascular Internal Medicine, Uji Tokushukai Hospital, Uji, Japan; ${ }^{7}$ Department of Pathology, Nara Medical University Hospital, Nara, Japan; ${ }^{8}$ Department of Pathology, Toshima Hospital, Tokyo, Japan; ${ }^{9}$ Pathology Division of Clinical Laboratory, National Center for Global Health and Medicine, Tokyo, Japan; ${ }^{10}$ Department of Pathology, Okinawa Prefectural Nanbu Medical Center and Children's Medical Center, Okinawa, Japan; ${ }^{11}$ Department of Pathology, Kyoto City Hospital, Kyoto, Japan; ${ }^{12}$ Tokyo Medical Examiner's Office, Tokyo, Japan; ${ }^{13}$ Department of Pathology, Hitachi Ltd. Hitachinaka General Hospital, Mito, Japan; ${ }^{14}$ Sakai City Institute of Public Health, Sakai, Japan; ${ }^{15}$ Department of Pathology and Laboratory Medicine, Odawara Municipal Hospital, Odawara, Japan; ${ }^{16}$ Department of Pediatrics, Oita Prefectural Hospital, Oita, Japan; ${ }^{17}$ Department of Emergency and Intensive Care, Narita Red Cross Hospital, Narita, Japan and ${ }^{18}$ Department of Pathology, Niigata Prefectural Central Hospital, Niigata, Japan

Twenty autopsy cases with 2009 pandemic influenza A (2009 H1N1) virus infection, performed between August 2009 and February 2010, were histopathologically analyzed. Hematoxylin-eosin staining, immunohistochemistry for type A influenza nucleoprotein antigen, and real-time reverse transcription-PCR assay for viral RNA were performed on formalin-fixed and paraffin-embedded specimens. In addition, the D222G amino acid substitution in influenza virus hemagglutinin, which binds to specific cell receptors, was analyzed in formalinfixed and paraffin-embedded trachea and lung sections by direct sequencing of PCR-amplified products. There were several histopathological patterns in the lung according to the most remarkable findings in each case: acute diffuse alveolar damage (DAD) with a hyaline membrane (four cases), organized DAD (one case), acute massive intra-alveolar edema with variable degrees of hemorrhage (three cases), neutrophilic bronchopneumonia (five cases) and tracheobronchitis with limited histopathological changes in alveoli (four cases). In two cases, the main findings were due to preexisting disease. Influenza virus antigen was only detected in the respiratory tract in $\mathbf{1 0}$ cases by immunohistochemistry. The antigen was detected in type II pneumocytes (three cases) in the epithelial cells of the trachea, bronchi and glands (six cases), and in the epithelial cells in both of the above (one case). The four cases with acute DAD presented with antigen-positive type II pneumocytes. In one case, the D222G substitution was detected in the lung as a major sequence, although 222D was prominent in the trachea, suggesting that selection of the viral clones occurred in the respiratory tract. In five

Correspondence: Dr N Nakajima, MD, PhD, Department of Pathology, National Institute of Infectious Diseases, 1-23-1 Toyama, Shinjyuku, Tokyo 162-8640, Japan.

E-mail: tenko@nih.go.jp

Received 6 April 2011; revised 24 June 2011; accepted 24 June 2011; published online 26 August 2011 


\begin{abstract}
cases, the pathogenesis of $2009 \mathrm{H} 1 \mathrm{~N} 1$ was confirmed to be viral infection in pneumocytes, which caused severe alveolar damage and fatal viral pneumonia. Further studies on both host and viral factors in autopsy or biopsy materials will be essential to elucidate the other pathogenic factors involved in influenza virus infection. Modern Pathology (2012) 25, 1-13; doi:10.1038/modpathol.2011.125; published online 26 August 2011
\end{abstract}

Keywords: autopsy; histopathology; immunohistochemistry; pathogenesis; real-time RT-PCR; D222G; $2009 \mathrm{H} 1 \mathrm{~N} 1$

Influenza virus causes respiratory infections with the potential for high morbidity and mortality, particularly in infants, the elderly, and those with underlying diseases. To control the seasonal epidemic of influenza virus infection, new vaccines are developed every year. Influenza virus also causes periodic pandemics. The emergence of avian H5N1 influenza virus infection in humans in 1997 alerted us the future outbreaks of new pandemic influenza virus infection.

In June 2009, the World Health Organization declared a novel influenza pandemic that entered the post-pandemic period in August 2010. ${ }^{1}$ Overall, the 2009 pandemic influenza A (2009 H1N1) virus did not cause particularly severe respiratory diseases, contrary to that expected at the start of the pandemic. In most cases, it caused subclinical infections or mild-to-moderate upper respiratory tract diseases. However, in some patients, it caused severe respiratory diseases, neurological complications, ${ }^{2-4}$ and myocardial symptoms., ${ }^{5,6}$ The 2009 H1N1 virus caused viral pneumonia with acute respiratory distress syndrome, ${ }^{7}$ which is not generally caused by seasonal influenza strains. It was also notable that younger people were affected with severe pneumonia, which was fatal in some cases. ${ }^{8,9}$

A total of 198 fatal cases with laboratory-confirmed 2009 H1N1 virus infection were reported by the Japanese Ministry of Health, Labour and Welfare, between August 2009 and March 2010. The fatality rate was estimated to be $0.001 \%$, which was much lower than that reported in other countries. ${ }^{10}$

Pathological studies with autopsy materials are essential to elucidate the pathogenesis of 2009 H1N1 virus infection. Autopsy findings in fatal cases of 2009 H1N1 have been reported from several countries. $^{11-15}$ The authors of the present study also reported the pathological and virological findings of two autopsy cases of 2009 H1N1 in Japan. ${ }^{16,17}$ Both cases revealed that the $2009 \mathrm{H} 1 \mathrm{~N} 1$ virus infected type II pneumocytes and caused diffuse alveolar damage (DAD).

In the present study, we evaluated various histopathological features of 20 cases with 2009 H1N1 virus infection, examined the distribution of influenza nucleoprotein antigens by immunohistochemistry, and quantified viral RNA extracted from formalin-fixed and paraffin-embedded tissue specimens. For the 10 cases with viral antigenand viral RNA-positive formalin-fixed and paraffinembedded tissue sections, we investigated amino acid substitutions at position 222 in hemagglutinin expressed by the $2009 \mathrm{H} 1 \mathrm{~N} 1$ virus. This position affects its receptor-binding specificity, and substitution from aspartic acid (D) to glycine (G) enables the virus to bind to the $\alpha 2-3$-linked sialic acid receptor $(\mathrm{SA} \alpha 2-3 \mathrm{Gal})$, which is expressed in distal bronchioles and type II pneumocytes. ${ }^{18-20}$

\section{Patients and methods}

\section{Patients}

A total of 20 fatal cases with $2009 \mathrm{H} 1 \mathrm{~N} 1$ infection were examined. Infection was confirmed by detecting viral RNA in nasopharyngeal swab specimens using the reverse-transcriptase PCR (RT-PCR) method at the first medical examination at each hospital. For six cases, the swabs were collected post mortem at the medical examiner's office. The autopsy tissue samples of the 20 fatal cases were sent from 15 hospitals to our laboratory for further pathological investigation between August 2009 and February 2010. Some tissue samples were formalinfixed and others were formalin-fixed and paraffinembedded. The tissue sections included the trachea $(n=15)$, lung $(n=20)$, heart $(n=10)$, brain $(n=5)$, liver $(n=5)$, spleen $(n=5)$, gastrointestinal tract $(n=5)$, kidney $(n=4)$, and lymph nodes $(n=3)$. This study was approved by the institutional medical ethical committee of the National Institute of Infectious Diseases, Japan (Approval No. 247).

\section{Histopathological Studies and Immunohistochemistry Assays}

Histopathological studies on formalin-fixed and paraffin-embedded specimens of all sections of 20 cases were performed using hematoxylin-eosin staining. Immunohistochemistry assays for type A influenza nucleoprotein antigen (InfA-NP) were performed to evaluate the distribution of $2009 \mathrm{H} 1 \mathrm{~N} 1$ as described previously. ${ }^{16}$ In brief, the sections were immunostained by the avidinbiotin complex immunoperoxidase method (LSAB2 kit/HRP/DAB, DAKO Cytomation, Copenhagen, Denmark) using a mouse monoclonal antibody against InfA-NP. ${ }^{21}$ For double-staining with a monoclonal antibody against cell type-specific marker proteins, a rabbit polyclonal antibody against InfANP was used. To characterize the virus-infected cells, confocal laser scanning microscopy was used to visualize double immunofluorescence staining for 
Table 1 Clinical information of the 20 autopsy cases confirmed 2009 H1N1 virus infection

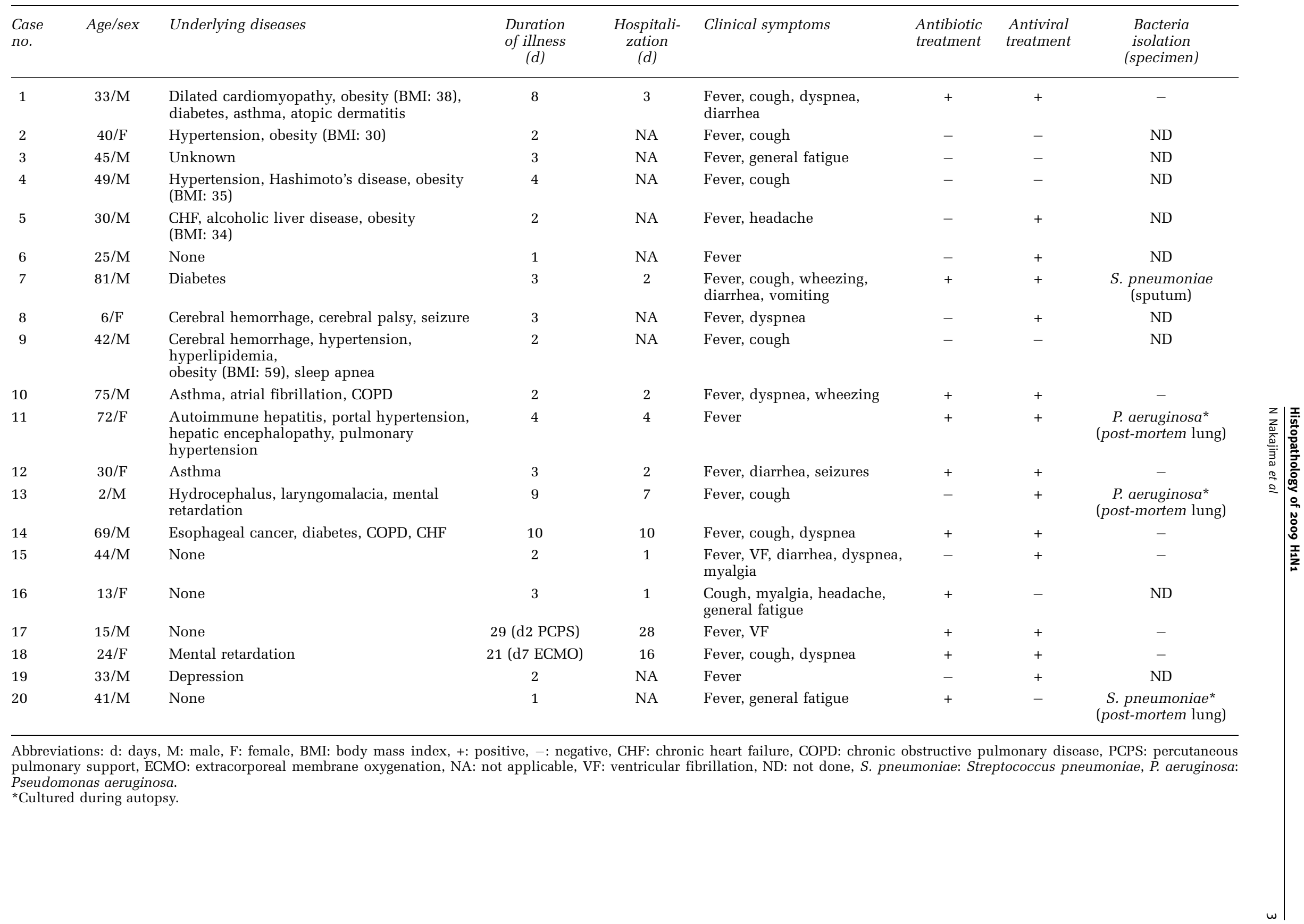


InfA-NP and for cell type-specific marker proteins, epithelial membrane antigen (EMA; epithelial cells), surfactant apoprotein D (SP-D; type II pneumocytes), cytokeratin AE1/AE3 (epithelial cells), CD68 (PG-M1; macrophages), and CD34 (endothelial cells), as previously described..$^{22}$ All mouse monoclonal antibodies, except for rabbit polyclonal antibody for SP-D, were purchased from DAKO Cytomation. Anti-SP-D antibody was purchased from Chemicon (Temecula, CA). Alexa Fluor 568-conjugated anti-mouse or anti-rabbit IgG (Molecular Probes, Eugene, OR), and Alexa Fluor 488conjugated anti-rabbit or anti-mouse IgG (Molecular Probes) were used as secondary antibodies.

\section{Nucleic Acid Extraction and Real-Time RT-PCR (rRT-PCR) Assays}

Nucleic acid extracts were prepared from the formalin-fixed and paraffin-embedded tissue sections $(10 \mu \mathrm{m} \times 3)$ of 20 cases using a PureLink FFPE RNA total isolation kit (Invitrogen, Carlsbad, CA). The copy number of 2009 H1N1 was determined by rRT-PCR using an Mx3005P (Stratagene, La Jolla, CA) to amplify a segment within the hemagglutinin region of the $2009 \mathrm{H} 1 \mathrm{~N} 1$ virus RNA. The amount of human $\beta$-actin mRNA in the DNasetreated RNA extracted from each section was also determined, and this internal reference gene was used to normalize the amount of RNA isolated from a specimen. To amplify 2009 H1N1-hemagglutinin region, forward (swH1N1-HA-F: 5'-CCCCATTGCA TTTGGGTAAA-3') and reverse (swH1N1-HA-R: $5^{\prime}$ TGGAGAGTGATTCACACTCTGGAT- $3^{\prime}$ ) primers were used with the labeled probe $5^{\prime}$-(FAM)AACATTG CTGGCTGGATCCTGGGA(TAMRA)- $3^{\prime} .^{16}$ To amplify human $\beta$-actin mRNA, forward (5'-TGAGCGCGGCT ACAGCTT- $3^{\prime}$ ) and reverse ( $5^{\prime}$-TCCTTAATGTCACG CACGATTT-3') primers were used with the labeled probe $5^{\prime}$-(FAM)ACCACCACGGCCGAGCGG (TAMRA) $-3^{\prime} .{ }^{23}$ The copy numbers of 2009 H1N1hemagglutinin/cell were calculated using the $\beta$-actin mRNA copy number, which was estimated to be 1500 copies/cell, as previously described. ${ }^{16}$

\section{Sequence Analysis of Hemagglutinin of the 2009 H1N1 Virus}

Using the RNA samples extracted from viral antigen- and viral RNA-positive formalin-fixed and paraffin-embedded tissue sections, we analyzed the hemagglutinin sequence of the $2009 \mathrm{H} 1 \mathrm{~N} 1$ virus. To determine amino acid substitution in the receptor binding site of the 2009 H1N1 virus, the hemagglutinin region (nt. 634-793) was amplified with QIAGEN OneStep RT-PCR (QIAGEN, Tokyo, Japan)

Table 2 Histopathological findings in respiratory tracts of 20 autopsy cases

\begin{tabular}{|c|c|c|c|c|c|c|c|c|c|c|c|c|c|c|c|c|c|c|c|c|}
\hline \multirow[t]{2}{*}{ Histopathological features } & \multicolumn{20}{|c|}{ Case number } \\
\hline & 1 & 2 & 3 & 4 & 5 & 6 & 7 & 8 & 9 & 10 & 11 & 12 & 13 & 14 & 15 & 16 & 17 & 18 & 19 & 20 \\
\hline
\end{tabular}

Trachea, bronchus

Desquamation of epithelium

Necrotizing tracheobronchitis

Inflammatory cell infiltration

Congestion

Hemorrhage

Submucosal gland

inflammation

Mucosal eosinophilia

Bronchiole, alveolus

Diffuse alveolar damage

Hyaline membrane formation

Intra-alveolar edema

Intra-alveolar hemorrhage

Congestion

Microthrombi

Inflammatory cell infiltration

Fibrosis

Type II cells hyperplasia

Bronchopneumonia with

neutrophils

Others

Heart failure cell

Emphysema

Plexiform pattern

$\begin{array}{cccccccccccccccccccc}1+ & 3+ & 3+ & 1+ & 3+ & 3+ & 3+ & 2+ & 2+ & 1+ & 1+ & 2+ & 2+ & - & 2+ & 2+ & - & - & 1+ & - \\ - & 2+ & - & - & 1+ & 1+ & 3+ & - & - & - & - & 1+ & - & - & 2+ & 1+ & - & - & - & - \\ 1+ & 3+ & 1+ & 1+ & 1+ & 2+ & 3+ & 2+ & 2+ & - & 2+ & 3+ & 2+ & - & 2+ & 2+ & 1+ & - & 2+ & 2+ \\ - & 2+ & 2+ & 3+ & 3+ & 2+ & 2+ & - & 3+ & 2+ & - & 1+ & 2+ & 1+ & - & 1+ & - & - & 3+ & 2+ \\ - & - & - & - & 1+ & 1+ & 1+ & 1+ & 1+ & - & - & 1+ & - & - & 1+ & - & - & - & 1+ & - \\ - & 3+ & 1+ & 1+ & 1+ & 1+ & 2+ & 1+ & 1+ & - & 1+ & 1+ & - & - & - & - & - & - & 1+ & 1+ \\ & & & & & & & & & & & & & & & & & & & \\ - & - & - & - & - & 1+ & - & 1+ & 2+ & 1+ & 1+ & 2+ & - & - & 3+ & - & - & - & - & - \\ & & & & & & & & & & & & & & & & & & & \\ & & & & & & & & & & & & & & & & & & & \\ 3+ & 3+ & 2+ & 2+ & - & - & - & - & - & - & 1+ & - & - & - & - & - & - & 3+ & - & - \\ 2+ & 3+ & 3+ & 1+ & - & - & - & - & - & - & 1+ & - & - & - & - & - & - & - & - & - \\ 2+ & 3+ & 3+ & 3+ & 3+ & 3+ & - & - & 1+ & - & & - & - & 2+ & - & - & 3+ & - & 3+ & 3+ \\ 1+ & 1+ & - & 1+ & - & 2+ & - & - & 1+ & - & 2+ & 2+ & 2+ & 1+ & - & - & 3+ & 1+ & 2+ & 2+ \\ 2+ & 1+ & 2+ & 3+ & 3+ & 3+ & - & - & 3+ & 1+ & - & - & - & 1+ & - & - & 1+ & - & 3+ & 3+ \\ 1+ & - & - & - & - & - & - & - & - & - & 1+ & - & - & 1+ & - & - & - & 1+ & - & - \\ 1+ & 3+ & 2+ & - & - & - & 1+ & 2+ & 1+ & - & 2+ & - & 2+ & - & - & - & 2+ & 1+ & - & - \\ 1+ & 3+ & 1+ & 1+ & - & - & 2+ & 2+ & - & - & 2+ & 2+ & 1+ & 2+ & - & - & 3+ & 3+ & - & - \\ 3+ & - & - & - & - & - & - & - & - & - & 2+ & - & 1+ & 2+ & - & - & 2+ & 3+ & - & - \\ - & - & - & - & - & - & 3+ & 3+ & - & - & 1+ & - & 3+ & - & - & - & - & - & - & 2+\end{array}$

No finding (-), mild (1+), moderate (2+), severe (3+). 
using the following primers: forward, 5'-GCAGATG CATATGTTTTTGTGGG- ${ }^{\prime}$; and reverse, $5^{\prime}$-GATTTC CAGTTGCTTCGAATG $-3^{\prime}$ ). ${ }^{18}$ The RT-PCR products were directly sequenced with an ABI Prism 3130 Genetic Analyzer (Applied Biosystems, Foster City, CA) using the PCR primers.

\section{Results}

\section{Patients}

The clinical characteristics of the 20 patients are presented in Table 1. Fourteen patients were male.
The median age of the patients was 39.8 years (range, 2-81 years). Thirteen (65\%) patients were aged between 15 and 59 years. Thirteen patients $(65 \%)$ died within 3 days after the onset of influenza symptoms. The mean duration of illness was 5.7 days (range, 1-29 days). The most frequent medical history or underlying disease was cardiovascular disease, which included dilated cardiomyopathy, hypertension, chronic heart failure, atrial fibrillation, and pulmonary hypertension. Five patients were obese with a body mass index exceeding $30 \mathrm{~kg} / \mathrm{m}^{2}$. In terms of clinical symptoms, most patients had fever and cough. Antiviral treatment

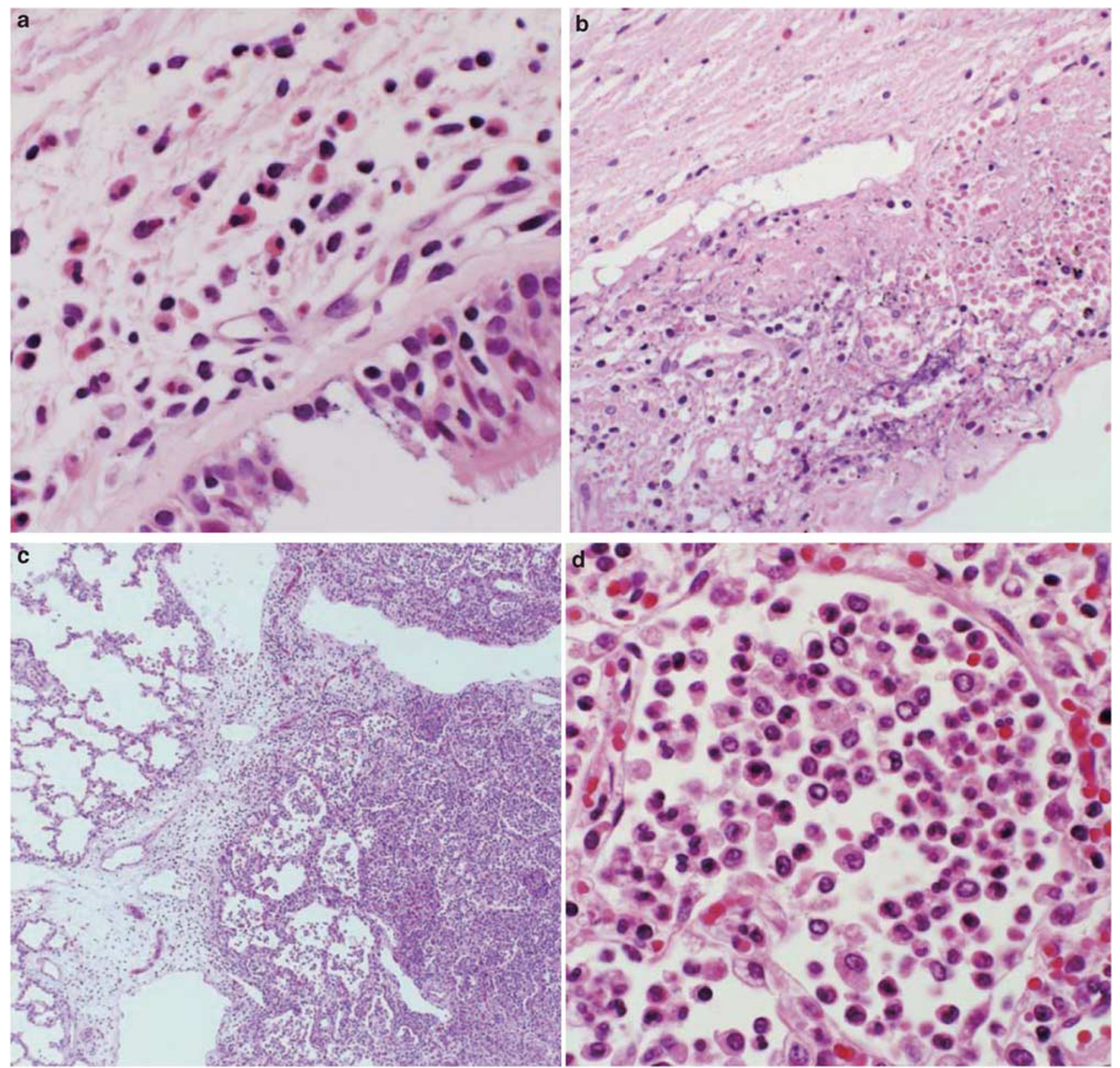

Figure 1 Representative histopathological findings on respiratory tissue sections with hematoxylin-eosin (H\&E) staining. (a) Mucosal infiltration of eosinophils and (b) necrotizing tracheobronchitis (case 15). (c and d) Acute bacterial bronchopneumonia with massive infiltration of neutrophils in the alveolar spaces (case 13). (e) Organized diffuse alveolar damage (DAD) with giant cells (inset) (case 18). (f) Acute massive alveolar edema and congestion (case 19). Original magnification, $\times 400$ : a, d, and $\mathbf{e}$ (inset); $\times 200: \mathbf{b}$ and $\mathbf{e}$; $\times 100: \mathbf{f} ; \times 40$ : $\mathbf{c}$. 


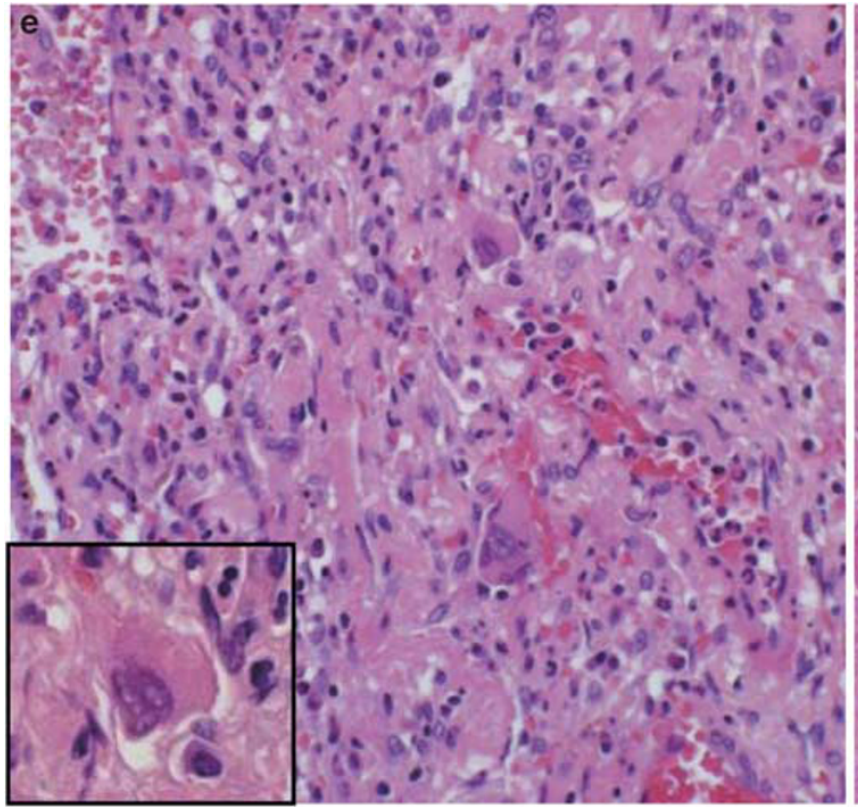

Figure 1 Continued.

was administered in 13 cases (oseltamivir in 11 cases and zanamivir in 2 cases) within $48 \mathrm{~h}$ after disease onset. Case 1 received oseltamivir from day 7 after disease onset. Six cases (cases 3-6, 19 and 20) from the medical examiner's office and cases 2 and 9 were found in the state of cardiac pulmonary arrest. Case 8 was dead on arrival without hospitalization. Eleven cases were hospitalized and seven of them were intubated because of hypoxia. Three cases (cases 15-17) were suspected of having fulminant myocarditis associated with influenza virus infection, because they suddenly developed ventricular fibrillation and cardiac arrest 2 or 3 days after disease onset. Bacterial culture was done for 11 cases. In three cases, lung specimens were taken aseptically during autopsy, and Streptococcus pneumoniae was cultured in one case and Pseudomonas aeruginosa in two cases. In one case, $S$. pneumoniae was cultured from the sputa on admission. The bacterial cultures were negative for the other seven cases.

\section{Histopathological Findings of the Trachea and Bronchi}

Histopathological changes in the trachea and bronchi are shown in Table 2. The most frequent histopathological findings were mucosal/submucosal mononuclear cell infiltration, multifocal desquamation of the epithelium, congestion and hemorrhage, mucosal infiltration of eosinophils (Figure 1a), and necrotizing tracheobronchitis (Figure 1b). We also observed inflammation in and around the bronchial glands (Figure 2e and k). The duration of illness in cases 17 and 18 was 29 and 21 days, respectively. The trachea and bronchi in

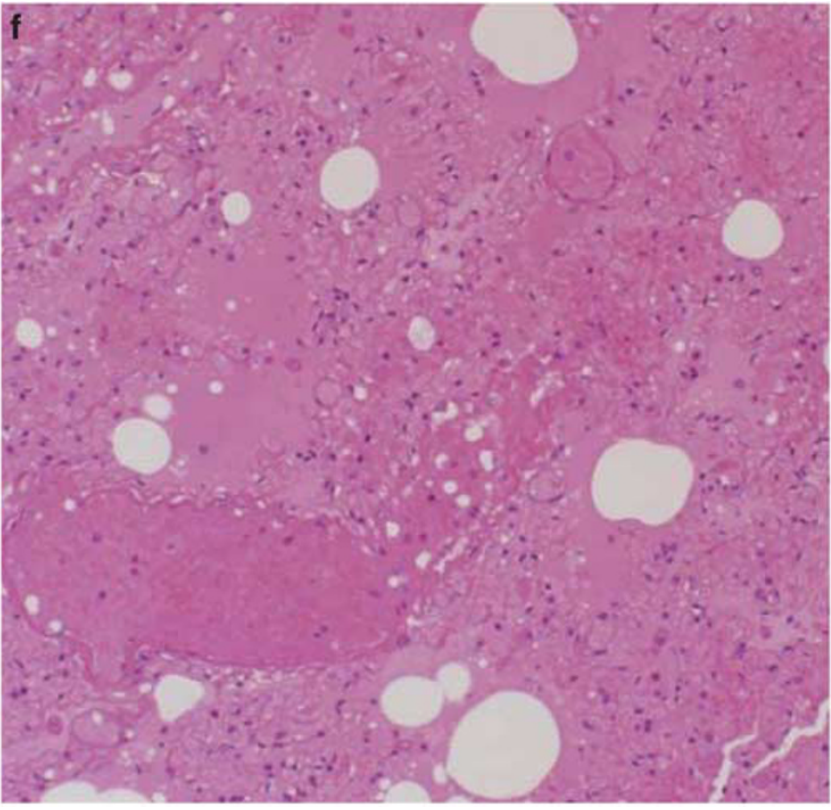

these cases were regenerated and presented no remarkable pathological changes.

\section{Histopathological Findings of the Lung}

Histopathological findings of the lung are shown in Table 2. Exudative or organized DAD, hyaline membrane formation, acute intra-alveolar edema, congestion and/or hemorrhage, capillary or small vessel thrombosis, interstitial or intra-alveolar inflammatory cell infiltration, fibrosis, and type II pneumocyte hyperplasia were observed (Figure 2a, $\mathrm{C}$, and g). Cases with massive infiltration of neutrophils in the alveolar spaces and bacterial isolation from a pharyngeal swab or lung specimens were defined as having secondary or coincident bacterial bronchopneumonia (Figure 1c and d). We excluded the pathological findings in case 17 from the pathological assessment of influenza infection, because they were extensively modified by complications caused by the long duration of percutaneous pulmonary support.

According to the most prominent histopathological feature in each case, there were several histopathological patterns. Five cases presented with DAD: acute DAD with a hyaline membrane (cases 1-4; Figure 2a, c, and g) and organized DAD (case 18; Figure 1e). Three cases had acute massive intraalveolar edema with variable degrees of hemorrhage (cases 5, 6, and 19; Figure 1f) and five cases had secondary or coincident bacterial pneumonia (cases 7, 8, 11, 13, and 20; Figure 1c and d). Four cases had more severe lesions in the upper airway with limited intra-alveolar edema and hemorrhage (cases 9 and 12), or no pathological changes in the lower airway (cases 15 and 16). The main histopathological 
findings in cases 10 and 14 included preexisting emphysema with congestion and edema.

\section{Histopathological Findings of Extrapulmonary Organs}

The pathological findings of heart tissue specimens in three cases, which were suspected of having myocarditis as described above, did not show inflammatory cell infiltration or myocyte necrosis. Cases 5 and 12 died of acute influenza virusassociated encephalopathy. In brain sections, case 5 showed histopathological changes in the brain, which included leakage of plasma proteins from vessels, activated microglia, and clasmatodendrosis of astrocytes (data not shown), whereas case 12 had
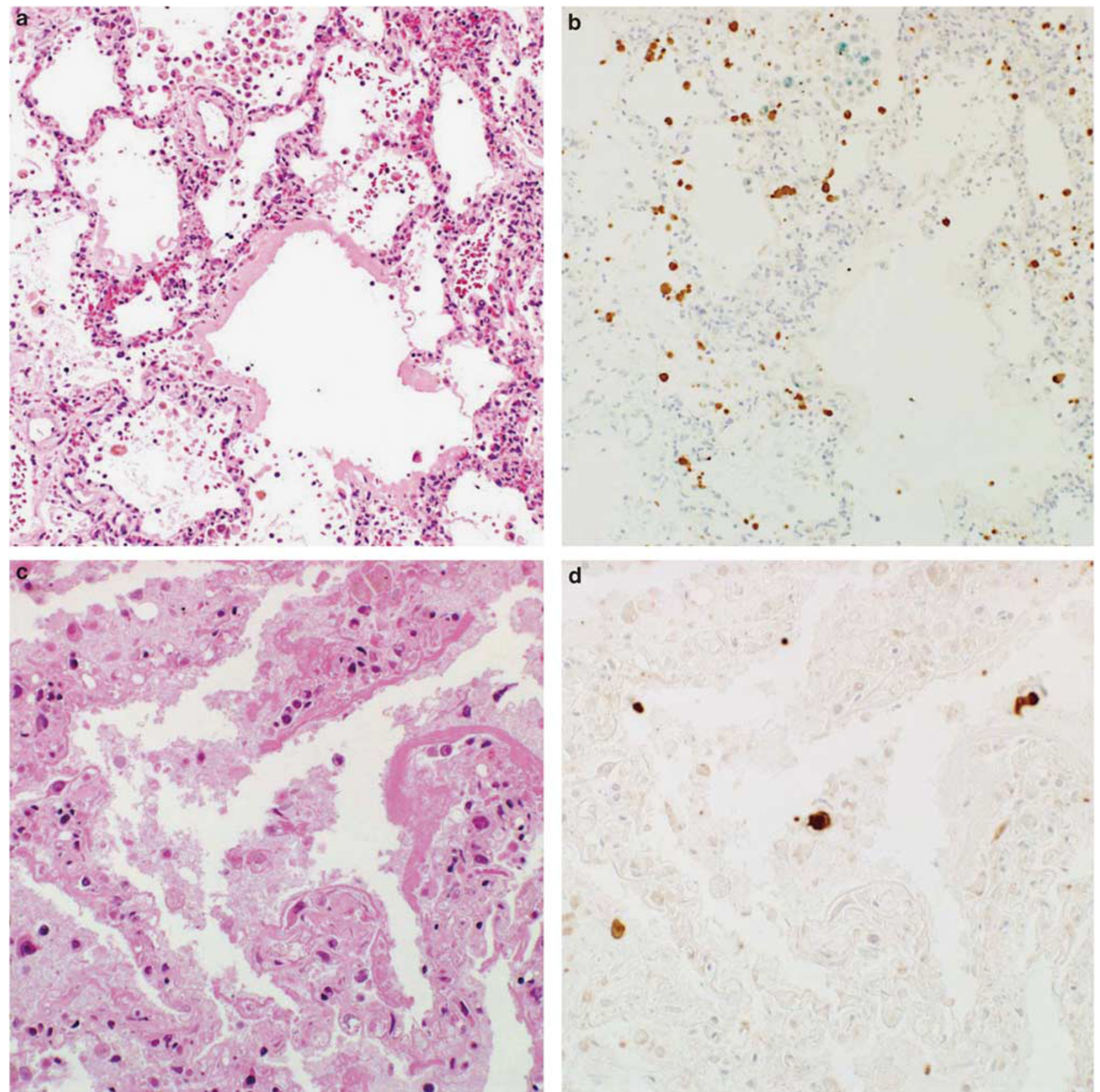

Figure 2 Hematoxylin-eosin (H\&E) staining (a, $, \mathbf{e}, \mathbf{g}, \mathbf{i}$, and $\mathbf{k}$ ) and immunohistochemistry for type A influenza nucleoprotein antigen (InfA-NP) (b, d, f, h, $\mathbf{j}$, and $\mathbf{l}$ ) using consecutive sections. Histopathological findings in the alveoli (a-d, $\mathbf{g}$, and $\mathbf{h}$ ), in the submucosal glands (e, f, $\mathbf{k}$, and $\mathbf{l}$ ), and in the trachea (i and $\mathbf{j}$ ) are shown. InfA-NP antigens were detected in the alveoli presenting with an exudative stage of diffuse alveolar damage (DAD) with hyaline membrane formation and hyperplasia of type II pneumocytes (a and $\mathbf{b}$, case 1; $\mathbf{c}$ and $\mathbf{d}$, case 3). InfA-NP antigens were detected in both submucosal glands (e and f), and desquamated alveolar epithelial cells in case 4 ( $\mathbf{g}$ and $\mathbf{h}$ ). In case 7, InfA-NP antigens were detected in the nuclei of epithelial cells of trachea with mucosal mononuclear cell infiltration (i and j). In case 9, mononuclear cell infiltration around the bronchial submucosal glands (k) and viral antigens in submucosal glands (l) are shown. Original magnification, $\times 400$ : e, $\mathbf{f}, \mathbf{i}$, and $\mathbf{j} ; \times 200: \mathbf{a}-\mathbf{d}, \mathbf{g}, \mathbf{h}, \mathbf{k}$, and $\mathbf{l}$. 

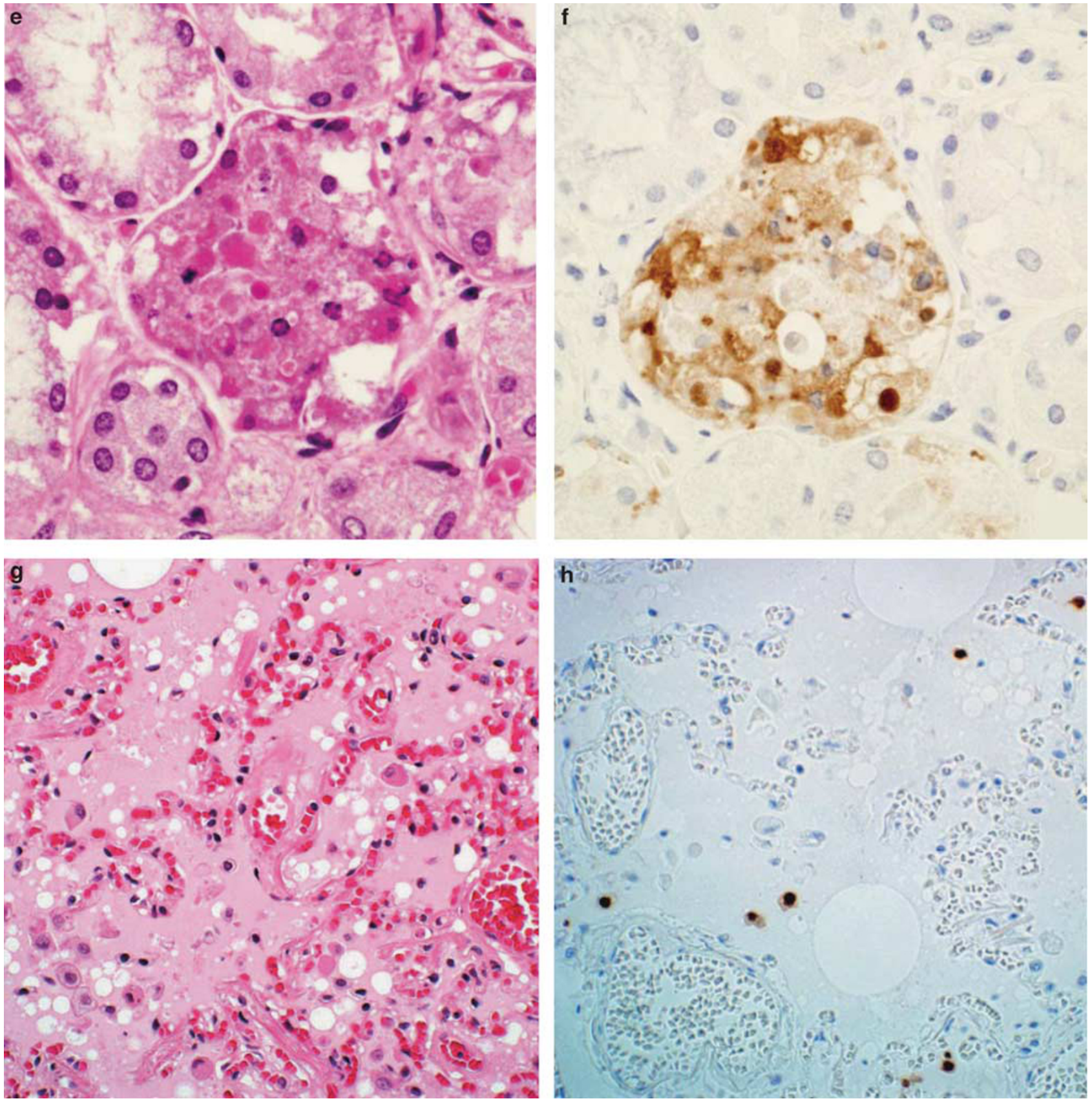

Figure 2 Continued.

gross brain edema and high plasma interleukin (IL)$6(16197 \mathrm{pg} / \mathrm{ml})$ and IL-10 (1156 pg/ml) concentrations, without remarkable histopathological changes in the brain.

\section{Detection of Viral Antigens and Viral RNA in Formalin-Fixed and Paraffin-Embedded Tissue Sections}

The histopathological findings described above are common in cases of seasonal influenza, ${ }^{24,25}$ past pandemic influenza, ${ }^{26,27}$ and $\mathrm{H} 5 \mathrm{~N} 1$ avian influenza. $^{22,28}$ However, these findings are not specific to influenza virus infection. Therefore, the distribution of virus antigen in formalin-fixed and paraffin-embedded tissue sections of 20 cases was examined by immunohistochemistry, with a monoclonal antibody for InfA-NP, and the relationship between histopathological findings and 2009 H1N1 virus infection was determined. InfA-NP antigen was only detected in the respiratory tract in 10 cases (cases 1-10). In cases 1-3, InfA-NP was detected in type II pneumocytes and in type I pneumocytes in the alveoli (Figure 2a-d). In case 4, it was detected in epithelial cells in the alveoli, bronchiole, glands, 

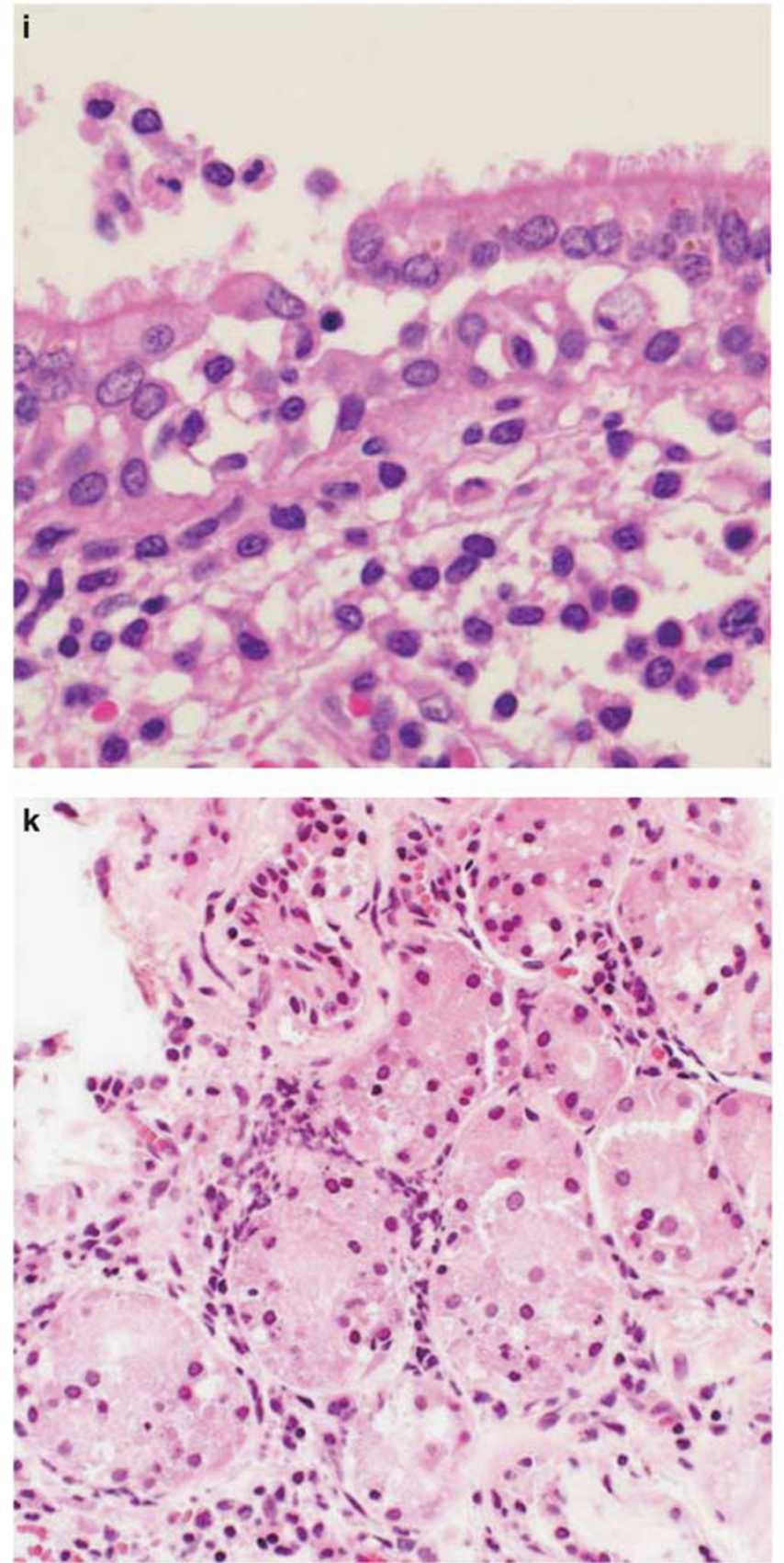

Figure 2 Continued.

bronchi, and tracheae (Figure 2e-h). In cases 5-10, InfA-NP was not detected in epithelial cells in the alveoli, but was detected in the submucosal glands, bronchi, and tracheae (Figure 2i-1). In cases 1, 2, and 8, InfA-NP was also detected in CD-68-positive alveolar macrophages as described below. Acute $\mathrm{DAD}$ is not specific for influenza virus infection, as it is caused by many factors; however, the detection of InfA-NP in alveolar epithelial cells appeared to be related to acute DAD with hyaline membrane formation (Table 3).

The 2009 H1N1 viral RNA in formalin-fixed and paraffin-embedded tissue sections was evaluated by
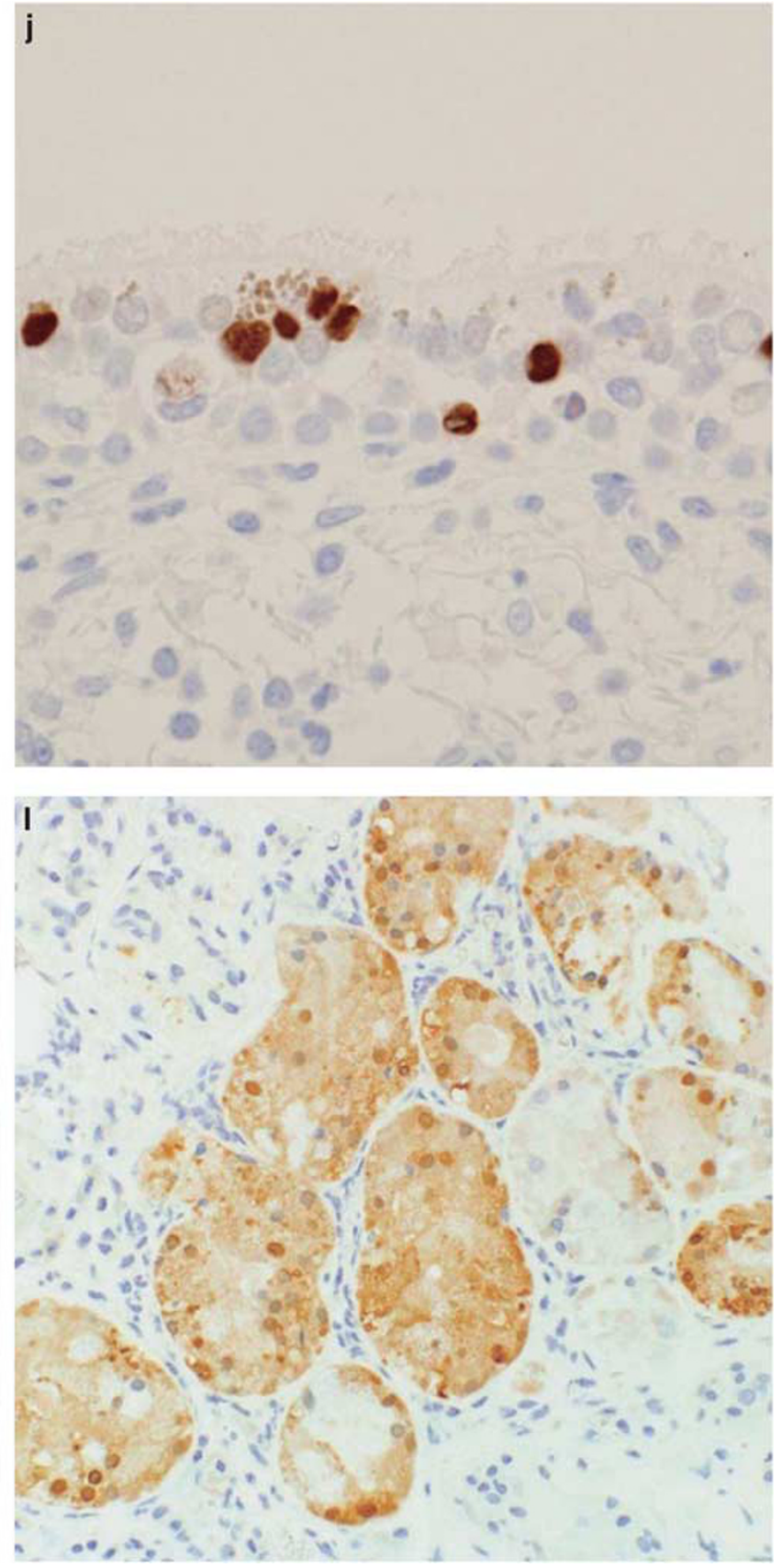

rRT-PCR. The relative virus RNA copy number per cell showed the quantitative distribution in each case. The results of rRT-PCR only show that viral RNA is present in the examined section. On the other hand, immunohistochemistry can identify the cells infected by the virus. Immunohistochemistry was more useful than the rRT-PCR method when RNA in the formalin-fixed and paraffin-embedded tissue sections had degenerated and little remained (ULD, Table 3). Viral RNA was detected from either the trachea or lung in 11 cases. RNA in formalinfixed and paraffin-embedded specimens in cases 3 and 6 was degenerated, and they were not available 
Table 3 Distribution of influenza nucleoprotein antigen and influenza virus RNA in formalin-fixed and paraffin-embedded trachea and lung sections

\begin{tabular}{|c|c|c|c|c|c|c|c|c|c|}
\hline \multirow[t]{3}{*}{$\begin{array}{l}\text { Case } \\
\text { no. }\end{array}$} & \multirow[t]{3}{*}{ Histopathological pattern } & \multicolumn{6}{|c|}{ Immunohistochemistry for influenza nucleoprotein antigen } & \multicolumn{2}{|c|}{$\begin{array}{c}\text { rRT-PCR } \\
(\text { copy/cell)** (a.a. 222)*** }\end{array}$} \\
\hline & & \multirow[t]{2}{*}{ Trachea } & \multicolumn{5}{|c|}{ Lung } & \multirow[t]{2}{*}{ Trachea } & \multirow[t]{2}{*}{ Lung* } \\
\hline & & & Bronchus & Gland & Bronchiolus & Alveolus & $\begin{array}{l}\text { Macro- } \\
\text { phage }\end{array}$ & & \\
\hline 1 & Acute DAD with HM & - & - & - & + & + & + & 0.65 (D) & $723(\mathrm{G})$ \\
\hline 2 & Acute DAD with HM & - & - & - & - & + & + & ULD & 12.8 (D) \\
\hline 3 & Acute DAD with HM & None & - & - & - & + & - & None & NA \\
\hline 4 & Acute DAD with HM & + & + & + & + & + & - & 14.8 (D) & 143 (D) \\
\hline 5 & $\begin{array}{l}\text { Massive pulmonary edema, } \\
\text { hemorrhage }\end{array}$ & - & - & + & - & - & - & 28.5 (D) & 30.9 (D) \\
\hline 6 & $\begin{array}{l}\text { Massive pulmonary edema, } \\
\text { hemorrhage }\end{array}$ & None & + & + & - & - & - & None & NA \\
\hline 7 & Bacterial pneumonia & + & + & + & - & - & - & 0.26 (D) & ULD \\
\hline 8 & Bacterial pneumonia & + & - & + & - & - & + & ULD & $35.5(\mathrm{D})$ \\
\hline 9 & $\begin{array}{l}\text { Hemorrhagic bronchitis, pulmonary } \\
\text { edema, congestion }\end{array}$ & + & + & + & - & - & - & 14.5 (D) & $22.3(\mathrm{D})$ \\
\hline 10 & Tracheobronchitis, emphysema & None & - & + & - & - & - & None & 37.8 (D) \\
\hline 11 & Bacterial pneumonia, plexiform pattern & None & - & - & - & - & - & None & 0.04 \\
\hline 12 & $\begin{array}{l}\text { Tracheobronchitis limited pulmonary } \\
\text { hemorrhage }\end{array}$ & - & - & - & - & - & - & 0.006 & 0.1 \\
\hline 13 & Bacterial pneumonia & - & - & - & - & - & - & 0.07 & 0.65 \\
\hline
\end{tabular}

Abbreviations: Case no.: case number, rRT-PCR: real-time reverse transcription-PCR assay, DAD: diffuse alveolar damage, HM: hyaline membrane, +: positive, -: negative, NA: not available due to RNA degeneration, none: no sample, ULD: under the limit of detection, D: aspartic acid, G: glycine. Lung*: lung section included bronchus, bronchiole, alveolus, macrophage or glands.

(Copy/cell)**: copy/cell $=2009 \mathrm{H} 1 \mathrm{~N} 1 / \beta$-actin $\times 1500$.

(a.a. 222)***: 222nd amino acid of hemagglutinin protein.

for analysis (NA, Table 3). Cases 14-20 are not included in Table 3, because neither viral antigens nor viral RNA were detected in the formalin-fixed and paraffin-embedded tissue sections.

\section{Identification of Influenza Nucleoprotein Antigen-Positive Cells}

InfA-NP-positive cells were identified. Double immunofluorescence staining for InfA-NP and for cell-type-specific marker proteins showed that InfA-NP signals were detected in EMA-positive epithelial cells, SP-D-positive type II pneumocytes, and CD68-positive alveolar macrophages. In the respiratory tract, they were detected in AE1/AE3positive epithelial cells of the trachea, bronchi, bronchioles, and submucosal glands (Figure 3). The InfA-NP signal was not detected in CD34-positive endothelial cells (data not shown).

\section{D222G Substitutions in the Hemagglutinin of 2009 H1N1 Virus}

The substitution from aspartic acid (D) to glycine (G) in the hemagglutinin protein enables the virus to bind to SA $\alpha 2-3 \mathrm{Gal}$, which is abundantly expressed in distal bronchioles and type II pneumocytes. ${ }^{18-20}$ The nucleotide sequence of the hemagglutinin gene in the 2009 H1N1 virus was examined by direct sequencing in formalin-fixed and paraffin- embedded trachea, and lung sections to detect amino acid substitutions at position 222 (225 in H3). The amino acid at position 222 in each section is shown in Table 3. The D222G substitution was only found in lung section specimens of case 1 . In addition, in case 1 , the results of direct sequencing suggested that there were several different 2009 H1N1 virus clones with different nucleic acids (Figure 4). When the sequences were translated to amino acids, the substitution from aspartic acid (D) to glycine (G) (D222G) was a major clone, whereas the substitution from aspartic acid (D) to asparagine $(\mathrm{N})(\mathrm{D} 222 \mathrm{~N})$ was a minor clone in the lung. This was consistent with the results of our previous analysis in frozen lung tissues using de novo sequencing with a next-generation DNA sequencer, ${ }^{29}$ an effective analytical tool, which needs a high number of copies of viral RNA for analysis. In this study, using formalin-fixed and paraffin-embedded specimens, and a direct sequencing method, we also detected different 2009 H1N1 virus clones with different nucleic acids in the tracheal sample from case 1 (Figure 4), where the D222G substitution was a minor clone and 222D was a major clone.

\section{Discussion}

Twenty autopsy cases in the present study were confirmed to have $2009 \mathrm{H} 1 \mathrm{~N} 1$ virus infection by 

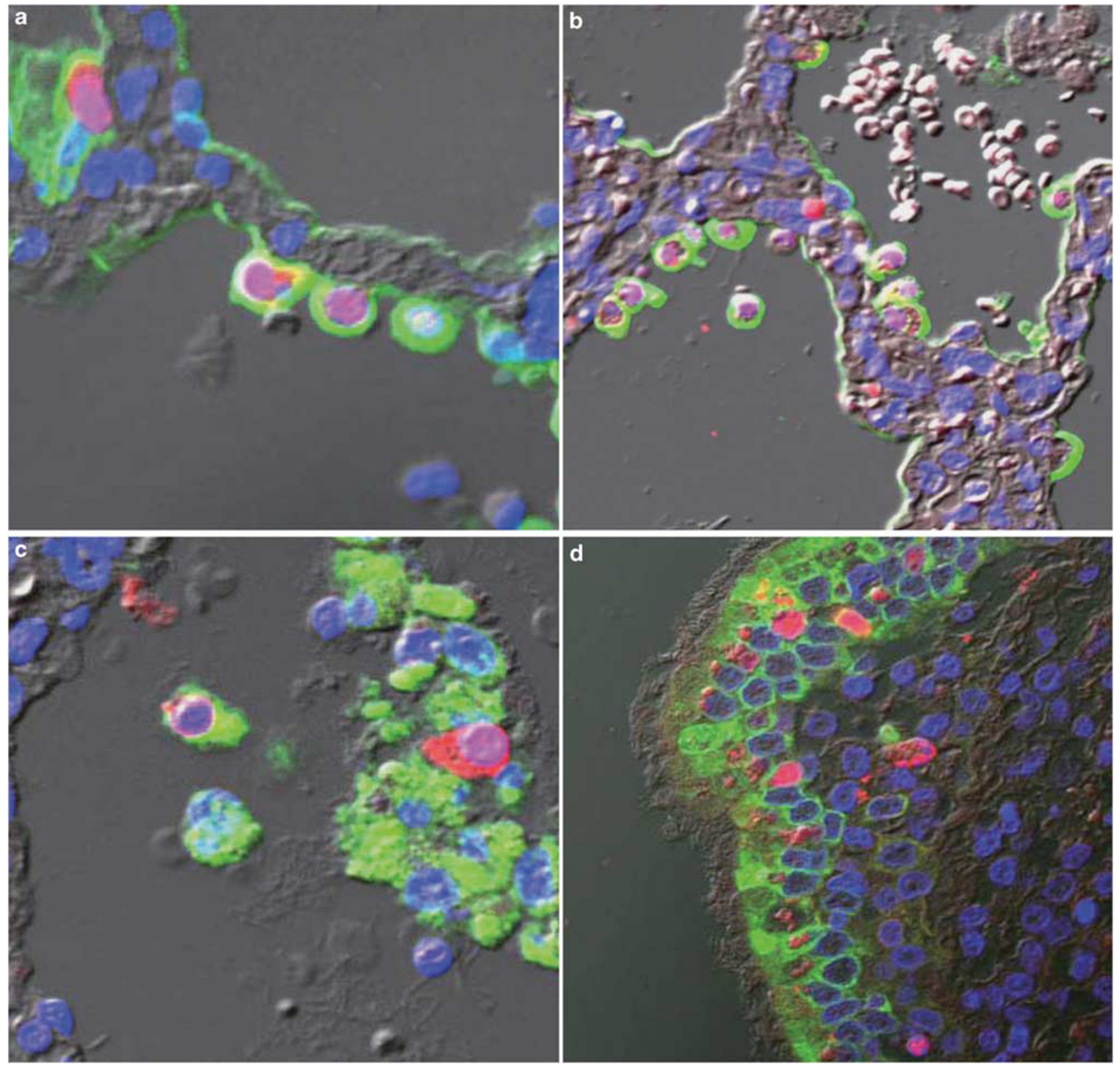

Figure 3 Double immunofluorescence staining. Type A influenza nucleoprotein antigen (InfA-NP; red) and cell-type-specific marker protein (green) are colocalized in the same cells. TO-PRO-3 nucleic acid staining (blue) and differential interference contrast (DIC) images are also shown. (a) InfA-NP (red) and epithelial membrane antigen (EMA) are shown in epithelial cells (green). (b) InfA-NP (red) and surfactant apoprotein D (SP-D) are shown in type II pneumocytes (green). (c) InfA-NP (red) and CD68 (PG-M1) are expressed in alveolar macrophages (green). The Inf-NP (red)-positive cell on the right is a detached type II pneumocyte. (d) InfA-NP (red) and cytokeratin AE1/ AE3 are shown in epithelial cells in the upper airways (green). Original magnification, $\times 400$.

RT-PCR from a nasopharyngeal swab. The characteristics of the patients were similar to those reported previously, ${ }^{11-15}$ except for a higher ratio of males, a lower prevalence of obesity, and a shorter duration of illness.

The 2009 H1N1 virus replicates in epithelial cells in both the respiratory tract in humans and in animal models. ${ }^{30}$ Potential infection in alveolar epithelial cells is the main feature that differentiates it from seasonal influenza strains. In the current study, autopsy cases of 2009 H1N1 infection presented with DAD with InfA-NP-positive alveolar epithelial cells, similar to those of avian H5N1 infection in humans. ${ }^{11-17,28}$ The first autopsy case in Japan (case 1) also showed that the 2009 H1N1 virus replicated in types I and II alveolar epithelial cells and caused fatal viral pneumonia. ${ }^{16}$ However, the present study with pathological analysis of 20 autopsy cases showed that fewer than expected cases showed similarities to case 1 . Only five (25\%) 


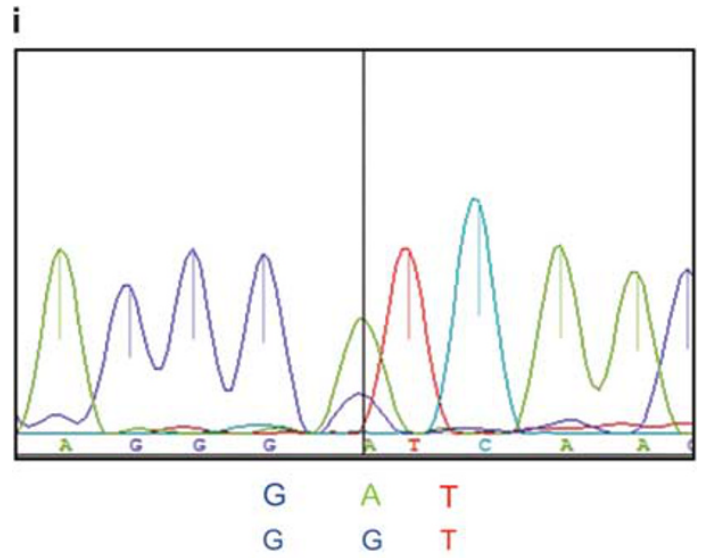

ii

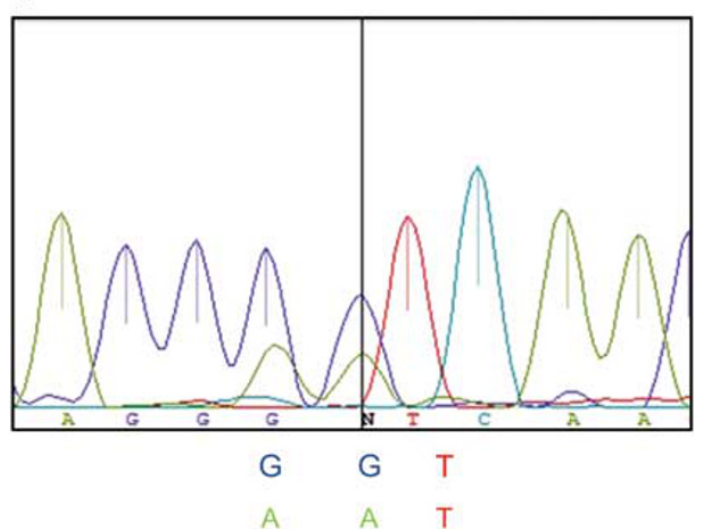

Figure 4 Amino acid residue at position 222 (225 in H3) in hemagglutinin of the 2009 pandemic influenza A (2009 H1N1) virus in formalin-fixed and paraffin-embedded trachea, and lung tissue sections was determined by direct sequencing. The nucleotide sequence around the amino acid residue at position 222 in the hemagglutinin gene of the $2009 \mathrm{H} 1 \mathrm{~N} 1$ virus in case 1 is shown. (i) GAT (D, aspartic acid) was the major sequence, and GGT (G, glycine) was the minor sequence in the trachea. (ii) GGT ( $\mathrm{G}$, glycine) was the major sequence and AAT (N, asparagine) was the minor sequence in the lung.

cases showed DAD histopathologically, which is much less than that reported in other countries. It was previously reported that $95-100 \%$ of cases with fatal 2009 H1N1 influenza showed a spectrum of histopathological changes of DAD. ${ }^{12-14}$ In addition, it is noteworthy that four $(20 \%)$ cases had no particular findings in the lower respiratory tract. The cause of death in at least six cases was not respiratory failure, and instead was encephalopathy (cases 5 and 12), cerebral hemorrhage (cases 9 and 18), and myocardial dysfunction (cases 15 and 16). Encephalopathy ${ }^{2-4}$ and myocardial disorders ${ }^{5,6}$ have been reported to be associated with 2009 H1N1 virus infection and seasonal influenza virus infection, although the pathogenesis of these diseases and their relationship to influenza virus infection have not yet been elucidated.

There are no histopathological findings specific to 2009 H1N1 influenza infection, such as a specific inclusion body. The detection of InfA-NP in tissue sections is evidence of influenza virus infection and specific findings. In the current study, in 10 cases $(50 \%)$, InfA-NP was detected in formalin-fixed and paraffin-embedded tissue sections of the respiratory tract (Table 1; cases 1-10). In the other 10 cases, there were several reasons why the antigen was not detected, such as effective antiviral treatment, low virus titer, clearance of viral antigens after a long duration of illness, and the condition of formalinfixed and paraffin-embedded samples.

In the present study, 10 InfA-NP positive cases were investigated further. In cases 1-4, InfA-NP was detected in alveolar epithelial cells. Common clinical features of these patients included obesity and no antiviral treatment within $48 \mathrm{~h}$ after disease onset (Table 1). In addition, they presented with histopathological findings of acute DAD, similar to H5N1 avian influenza virus infection in humans ${ }^{28}$ (Table 2). The distribution of InfA-NP in cases 5-10 was similar to that observed in seasonal influenza virus infection. ${ }^{25}$ Their histopathological findings and the causes of death, which included secondary bacterial pneumonia, were also similar to those of seasonal influenza (Table 2).

Seasonal influenza virus binds to SA $\alpha 2-6 \mathrm{Gal}$, which is expressed in epithelial cells in the upper respiratory tract. The avian influenza virus preferentially binds to SA $\alpha 2-3 \mathrm{Gal}$, which is expressed in distal bronchioles and type II pneumocytes in the lower respiratory tract. ${ }^{31}$ It was shown in vitro that the $2009 \mathrm{H} 1 \mathrm{~N} 1$ virus binds to both SA $\alpha 2-6 \mathrm{Gal}$ and SA $\alpha 2-3 \mathrm{Gal}^{32}$ In this study, cases 1-4 presented with DAD with InfA-NP-positive pneumocytes. We expected to find the D222G substitution in hemagglutinin gene recovered from lung specimens in these cases, but it was only detected in case 1 . In case 1, $2009 \mathrm{H} 1 \mathrm{~N} 1$ presented with a quasispecies at position 222 in the lung and in the trachea, and the major sequence was different in each location (Figure 4). The D222G clone, which was a minor clone in the trachea, propagated and accumulated in the lung where SA $\alpha 2-3 \mathrm{Gal}$ is abundantly expressed. In the other cases, quasispecies were not found by direct sequencing and the amino acid at position 222 was aspartic acid. The $222 \mathrm{D}$ clone rarely binds to $\mathrm{SA} \alpha 2-3 \mathrm{Gal}$, according to the results of an in vitro study. ${ }^{33}$ This might explain why many more InfANP antigens were detected in pneumocytes in case 1 than in cases 2 and 4.

\section{Acknowledgements}

We thank Drs M Watanabe, A Takiyama, Y Urata, H Miwa, T Okamura, and K Nakanishi for clinical and pathological information on the patients. This work was supported by a Health and Labour Sciences Research Grant on Emerging and Re-emerging Infectious Diseases (H22 ShinkoIppan-014), from the Ministry of Health, Labour and Welfare, Japan. 


\section{Disclosure/conflict of interest}

The authors declare no conflict of interest.

\section{References}

1 Dawood FS, Jain S, Finelli L, et al. Emergence of a novel swine-origin influenza A (H1N1) virus in humans. N Engl J Med 2009;360:2605-2615.

2 Ichiyama $\mathrm{T}$, Isumi $\mathrm{H}$, Ozawa $\mathrm{H}$, et al. Cerebrospinal fluid and serum levels of cytokines and soluble tumor necrosis factor receptor in influenza virus associated encephalopathy. Scand J Infect Dis 2003;35:59-61.

3 Togashi T, Matsuzono Y, Narita M, et al. Influenzaassociated acute encephalopathy in Japanese children in 1994-2002. Virus Res 2004;103:75-78.

4 Moon SM, Kim SH, Jeong MH, et al. Acute encephalopathy and pandemic (H1N1) 2009. Emerg Infect Dis 2010;16:1811-1813.

5 Bratincsak A, El-Said HG, Bradley JS, et al. Fulminant myocarditis associated with pandemic H1N1 influenza A virus in children. J Am Coll Cardiol 2010;55: 928-929.

6 Ukimura A, Izumi T, Matsumori A. A national survey on myocarditis associated with the 2009 influenza A (H1N1) pandemic in Japan. Circ J 2010;74: 2193-2199.

7 Perez-Padilla R, de la Rosa-Zamboni D, Ponce de Leon S, et al. Pneumonia and respiratory failure from avian-origin influenza A (H1N1) in Mexico. N Engl J Med 2009;361:680-689.

8 Lee EH, Wu C, Lee EU, et al. Fatalities associated with the $2009 \mathrm{H} 1 \mathrm{~N} 1$ influenza A virus in New York city. Clin Infect Dis 2010;50:1498-1504.

9 Centers for Disease Control and Prevention. CDC estimates of $2009 \mathrm{H} 1 \mathrm{~N} 1$ influenza cases, hospitalizations and deaths in the United States, April 2009March 13, 2010. Available from http://www.cdc.gov/ h1n1flu/estimates/April_March_13.htm.

10 Centers for Disease Control Prevention. Deaths and hospitalizations related to 2009 pandemic influenza A (H1N1)-Greece, May 2009-February 2010. (MMWR) Morb Mortal Wkly Rep 2010;59:682-686.

11 Soto-Abraham MV, Soriano-Rosas J, Diaz-Quinonez A, et al. Pathological changes associated with 2009 H1N1 virus. N Engl J Med 2009;361:2001-2003.

12 Maud T, Hajjar LA, Callegari GD, et al. Lung pathology in fatal novel human influenza A (H1N1) infection. Am J Respir Crit Care Med 2010;181:72-79.

13 Gill JR, Sheng ZM, Ely SF, et al. Pulmonary pathologic findings of fatal 2009 pandemic influenza A /H1N1 viral infections. Arch Pathol Lab Med 2010;134: 235-243.

14 Shieh WJ, Blau DM, Denison AM, et al. 2009 pandemic influenza A (H1N1): pathology and pathogenesis of 100 fatal cases in the United States. Am J Pathol 2010;177:166-175.

15 Rosen DG, Lopez AE, Anzalone ML, et al. Postmortem findings in eight cases of influenza A/H1N1. Modern Pathol 2010;148:1-9.

16 Nakajima N, Hata S, Sato Y, et al. The first case of pandemic influenza (A/H1N1) virus infection in Japan: detection of a high copy number of the virus in type II alveolar epithelial cells by pathological and virological examination. Jpn J Infect Dis 2010;63:67-71.

17 Takiyama A, Wang L, Tanino M, et al. Sudden death of a patient with pandemic influenza (A/H1N1pdm) virus infection by acute respiratory distress syndrome. Jpn J Infect Dis 2010;63:72-74.

18 Chen H, Wen X, To KKW, et al. Quasispecies of the D225G substitution in the hemagglutinin of pandemic influenza A(H1N1) 2009 virus from patients with severe disease in Hong Kong, China. J Infect Dis 2010; 201:1517-1521.

19 World Health Organization. Preliminary review of D222G amino acid substitution in the haemagglutinin of pandemic influenza A (H1N1) 2009 viruses. Wkly Epidemiol Rec 2010;85:21-22.

20 Chutinimikul S, Herfst S, Steel J, et al. Virulenceassociated substitution D222G in hemagglutinin of 2009 pandemic influenza A (H1N1) virus affects receptor binding. J Virol 2010;84:11802-11813.

21 Chen Z, Sahashi Y, Matsuo K, et al. Comparison of the ability of viral protein- expressing plasmid DNAs to protect against influenza. Vaccine 1998;16:1544-1549.

22 Liem NT, Nakajima N, Phat LP, et al. H5N1-infected cells in lung with diffuse alveolar damage in exudative phase from a fatal case in Vietnam. Jpn J Infect Dis 2008;61:157-160.

23 Kuramochi H, Hayashi K, Uchida K, et al. Vascular endothelial growth factor messenger RNA expression level is preserved in liver metastases compared with corresponding primary colorectal cancer. Clin Cancer Res 2006;12:29-33.

24 Guarner J, Paddock CD, Shieh WJ, et al. Histopathologic and immunohistochemical features of fatal influenza virus infection in children during the 20032004 season. Clin Infect Dis 2006;43:132-140.

25 Guarner J, Shieh WJ, Dawson J, et al. Immunohistochemical and in situ hybridization studies of influenza A virus infection in human lungs. Am J Clin Pathol 2000;114:227-233.

26 Taubenberger JK, Morens DM. The pathology of influenza virus infections. Annu Rev Pathol 2008;3: 499-522.

27 Kuiken T, Taubenberger J. Pathology of human influenza revisited. Vaccine 2008;26(Suppl 4):D59-D66.

28 Korteweg C, Gu J. Pathology, molecular biology, and pathogenesis of avian influenza A (H5N1) infection in humans. Am J Pathol 2008;172:1155-1170.

29 Kuroda M, Katano H, Nakajima N, et al. Characterization of quasispecies of pandemic 2009 influenza A virus (A/H1N1/2009) by de novo sequencing using a next-generation DNA sequencer. PLoS One 2010; 5:e10256

30 Itoh $\mathrm{Y}$, Shinya $\mathrm{K}$, Kiso $\mathrm{M}$, et al. In vitro and in vivo characterization of new swine-origin H1N1 influenza viruses. Nature 2009;460:1021-1025.

31 Shinya K, Ebina M, Yamada S, et al. Avian flu: influenza receptors in the human airway. Nature 2006; 440:435-436.

32 Childs RA, AS Palma S, Wharton T, et al. Receptorbinding specificity of pandemic influenza A (H1N1)2009 virus determined by carbohydrate microarray. Nat Biotechnol 2009;27:797-799.

33 Liu Y, Childs RA, Matrosovich T, et al. Altered receptor specificity and cell tropism of D222G haemoagglutinin mutants from fatal cases of pandemic A (H1N1) 2009 influenza. J Virol 2010;84:12069-12012. 